LAWRENCE LIVERMORE N A T IO N A L LABORATORY
Simulation of Turbulence in the Divertor Region of Tokamak Edge Plasma

M.V. Umansky, T.D. Rognlien, X.Q. Xu

October 5, 2004

Journal of Nuclear Materials 
This document was prepared as an account of work sponsored by an agency of the United States Government. Neither the United States Government nor the University of California nor any of their employees, makes any warranty, express or implied, or assumes any legal liability or responsibility for the accuracy, completeness, or usefulness of any information, apparatus, product, or process disclosed, or represents that its use would not infringe privately owned rights. Reference herein to any specific commercial product, process, or service by trade name, trademark, manufacturer, or otherwise, does not necessarily constitute or imply its endorsement, recommendation, or favoring by the United States Government or the University of California. The views and opinions of authors expressed herein do not necessarily state or reflect those of the United States Government or the University of California, and shall not be used for advertising or product endorsement purposes. 


\title{
Simulation of Turbulence in the Divertor Region of Tokamak Edge Plasma
}

\author{
M.V.Umansky, T.D.Rognlien, and X.Q.Xu, \\ Lawrence Livermore National Laboratory, \\ Livermore, CA 94550, USA
}

July 23, 2004

\begin{abstract}
Results are presented for turbulence simulations with the fluid edge turbulence code BOUT [1]. The present study is focussed on turbulence in the divertor leg region and on the role of the X-point in the structure of turbulence. Results of the present calculations indicate that the ballooning effects are important for the divertor fluctuations. The X-point shear leads to weak correlation of turbulence across the $\mathrm{X}$-point regions, in particular for large toroidal wavenumber. For the saturated amplitudes of the divertor region turbulence it is found that amplitudes of density fluctuations are roughly proportional to the local density of the background plasma. The amplitudes of electron temperature and electric potential fluctuations are roughly proportional to the local electron temperature of the background plasma.

JNM keywords

T0100

PSI-16 keywords

Edge modeling PACS keywords

Corresponding (and presenting) author: M.V.Umansky

Address: L-630, LLNL, 7000 East Ave, P.O. Box 808, Livermore, CA 94551

E-mail: umansky@llnl.gov 


\section{Introduction}

Turbulence in the tokamak boundary plasma is a challenging and fundamentally important problem for tokamak physics. The turbulent plasma transport in the edge is known to strongly affect the global energy confinement time and hence the overall performance of the tokamak. On the other hand, the level of edge plasma transport determines the width of the power deposition profiles on the material plasma facing components.

It has been long recognized that the presence of the X-point has a crucial importance for tokamak edge turbulence [2]. Near the X-point the poloidal field, $B_{\theta}$, is small, which leads to a large poloidal wavenumber, $k_{\theta}$. Also, due to the strong shearing of the magnetic field near the X-point, the flux tubes are squeezed radially, which leads to a large radial wavenumber, $k_{r}$. Both effects lead to strongly increasing perpendicular wave numbers near the Xpoint. This greatly enhances the effects of plasma resistivity there, causing decoupling of flute-like perturbations across the X-point region. Numerical calculations of linear resistive ballooning modes in the edge plasma in the realistic X-point geometry demonstrated that the presence of the X-point leads to disconnection of eigenmodes across the X-point [3]. Similar conclusions were obtained in non-linear simulations of edge plasma turbulence [4].

In limiter tokamak configurations, edge plasma is supported by conducting end walls. In such configurations emerge sheath-driven instabilities $[5,6,2,7]$. These instabilities are driven by interplay of radial temperature gradients and the sheath boundary conditions on the end walls, but curvature effects can also be important.

Recently, it has been recognized that conducting-wall type instability can exist in the X-point tokamak configuration, where it is confined to the divertor legs by the X-point shear effects [8]. The analysis in [8] was limited to the linear instability. Recent non-linear simulations of the the divertor leg instability with the BOUT code [1] were described in our previous paper [9]. Here we continue and extend the analysis presented in [9]. 


\section{Simulations of edge turbulence with BOUT}

\subsection{Physics model}

BOUT $[1,4]$ is a $3-\mathrm{D}$ turbulence code solving a system of plasma fluid equations based on the reduced Braginskii equations [10]. These are equations for plasma density, $n_{i}$, electron and ion temperatures, $T_{e, i}$, electron and ion parallel velocity, $V_{\| e, i}$, electrostatic potential, $\phi$, and the vector potential, $A_{\|}$. BOUT uses the quasi-ballooning coordinates [11] to efficiently handle the realistic geometry of tokamak. BOUT has been applied to several tokamak experiments with encouraging similarity to experimental spatial and temporal spectra of edge plasma fluctuations $[12,13,14]$.

\subsection{Average profile evolution}

Starting from initial plasma profiles, BOUT can be used in the "predictive" mode, when the toroidally average plasma profiles are evolved. However, BOUT does not have all physics that, aside from the turbulent transport, is involved in forming the steady state plasma profiles, i.e., the atomic physics, neutral transport, impurity transport and radiation. Therefore, the full timeevolution with BOUT results in steady-state plasma profiles that, in general, don't match the experimental ones.

To circumvent this problem, BOUT can be run in the "interpretive" mode when the toroidally average plasma profiles are frozen. The latter can be thought of as adding to the equations fake source terms that are functions of space but not of plasma variables, so that the equilibrium is maintained but stability is not affected. For the present study we use frozen plasma profiles, as described in detail further.

\section{$3 \quad$ Background plasma profiles}

In most of previous studies with $\operatorname{BOUT}[4,12,15,16]$, the poloidal variation of background plasma parameters was not taken into account. However, the ratio of conditions at the mid-plane and in the divertor, $T_{\text {mid }} / T_{d i v} \sim$ $n_{\text {div }} / n_{\text {mid }}$, can be on the order of 3-10 in the high-recycling regime [17].

For the present study, we use a set of analytic background plasma profiles that approximate the equilibrium state of the edge plasma as known from 
experimental data and edge transport simulations with the UEDGE code [18]. Figure 1 illustrates the background profiles of $n_{i 0}$ and $T_{e 0}$ for poloidally constant (top) and poloidally variable (bottom) cases. The radial mid-plane profiles of $n_{i 0}$ and $T_{e 0}$ are taken exponential with e-folding length typical for a tokamak. For the poloidal variation of $T_{e 0}$, we use the analytic expression

$$
T_{e 0}(s)=\left[T_{d i v}^{\gamma}+4 s(1-s)\left(T_{m i d}^{\gamma}-T_{d i v}^{\gamma}\right)\right]^{1 / \gamma} .
$$

Here $\mathrm{s}$ is the poloidal coordinate measured from the inner plate to the outer one with the total distance normalized to unity, $T_{d i v}$ is the divertor temperature, $T_{\text {mid }}$ is the mid-plane temperature, and the constant factor $\gamma$ is typically chosen in the range 2-3 which provides a good fit to typical UEDGE calculations. The poloidal variation of $n_{i 0}$ is set to keep plasma pressure constant along the field line: $n_{i 0}\left(T_{e 0}+T_{i 0}\right)=$ const. The plasma flow velocity, $V_{i \| 0}$, and the DC (toroidally average) electric potential are set zero. The magnetic geometry corresponds to a typical DIII-D shot with a lower single null.

\section{Results of simulations}

\subsection{Linear growth}

For the initial ramp-up stage of instability we compare the growth rates for fluctuations amplitude. Figure 2 is a plot of root-mean-square (RMS) average

1 of the fluctuating potential, $\tilde{\phi}$, against time, for separatrix locations at the inner leg, outer leg and the outer midplane. The figure shows that the growth rate in the divertor is much larger than that at the outer mid-plane. In part this can be explained by the larger plasma resistivity in the divertor, compared to that at the mid-plane, which gives a larger growth rate for a resistive-ballooning type instability.

Figure 3 shows the spatial distribution of the fluctuations amplitude during the initial ramp-up. Note that in the outer leg the fluctuations tend to grow outside of the separatrix, while in the inner leg they grow in the privateflux region. For the chosen profiles of the background plasma density and temperature these are the regions of unfavorable curvature for ballooning instability, which also points to the ballooning mechanism of the divertor instability.

\footnotetext{
${ }^{1}$ RMS average is defined as $\sqrt{<f^{2}>_{\zeta}}$, where $<>_{\zeta}$ means average over the toroidal coordinate.
} 


\subsection{Scaling trends for saturated turbulence}

Scaling trends for saturated turbulence amplitudes are analyzed using a set of BOUT runs with same radial profiles of $n_{i 0}, T_{e, i 0}$ upstream but varying $n_{i 0}, T_{e, i 0}$ in the divertor. Figure 4 shows a plot of $\tilde{n}_{i}$ against $n_{i 0}$, and Fig. 5 shows a plot of $\tilde{T}_{e}$ against $T_{e 0}$, and $\tilde{\phi}$ against $T_{e 0}$. The data in the figures are shown for the inner leg, in the outer leg the trends are similar. The results plotted in the figures show that the amplitudes of saturated $\tilde{n}_{i}$ fluctuations in the divertor are roughly proportional to the local $n_{i 0}$, while the amplitudes of $\tilde{\phi}$ and $\tilde{T}_{e}$ are roughly proportional to the local $T_{e 0}$.

\subsection{Spatio-temporal correlation for saturated turbu- lence}

To address the role of the X-point for these simulations we calculate the cross-correlation function for the turbulent potential using the data analysis package GKV [19]. The cross-correlation function (CCF) is defined as follows:

$$
C(\tilde{\phi}, \tilde{\phi})=\frac{<\tilde{\phi}\left(r_{0}, \theta, \zeta+\Delta \zeta, t+\tau\right) \tilde{\phi}\left(r_{0}, \theta_{r e f}, \zeta, t\right)>_{\zeta, t}}{<\left|\tilde{\phi}\left(r_{0}, \theta_{r e f}, \zeta, t\right)\right|^{2}>_{\zeta, t}}
$$

Here $r_{0}$ is the radial index of the chosen flux surface, $\zeta$ is the toroidal grid index, and $\theta$ is the poloidal index. Also, $\tau$ is the time lag, $\theta_{r e f}$ is the reference poloidal index, $\left\langle>_{\zeta, t}\right.$ means averaging in $\zeta$ and $t$. The CCF measures similarity between the time evolution of fluctuating signals at the points $\left(r_{0}, \theta, \zeta\right)$ and $\left(r_{0}, \theta_{\text {ref }}, \zeta+\Delta \zeta\right)$, averaged over time and $\zeta$. Note that $\zeta$ is also a magnetic line label in BOUT coordinates, so $\Delta \zeta \approx 0$ for nearby magnetic lines.

As discussed in [9], the CCF for the turbulent potential, Eq. (2), is negligibly small except for $\Delta \zeta \approx 0$. This means that fluctuations on non-adjacent magnetic lines are not physically connected. On the other hand, for $\Delta \zeta \approx$ 0 , the CCF is large only if $\theta$ and $\theta_{\text {ref }}$ are not separated by an X-point. This means that for points on the same magnetic line fluctuations become physically disconnected by X-points.

This is illustrated by Fig. 6, where the CCF, Eq. (2), is shown for a flux surface just outside of the separatrix, with $\theta_{\text {ref }}$ at the outer mid-plane, with $\Delta \zeta=0$ and $\tau=0$, for three different toroidal wave numbers, $k_{\phi}$. The figure shows that the CCF has cutoffs near both the lower X-point and the upper $\mathrm{X}$-point regions, and the cutoff is more pronounced for larger $k_{\phi}$, as expected 
from the theoretical analysis [20]. Note that the upper X-point is not in the simulation domain but still it manifests itself via the stronger magnetic field shear.

\section{$5 \quad$ Summary and conclusions}

We have conducted numerical simulations of turbulence in the divertor region of tokamak with the BOUT code. The results of the present calculations show that the ballooning drive is important during the linear ramp-up of divertor fluctuations. For saturated turbulence, the amplitude of density fluctuations in the divertor is roughly proportional to the background plasma density, while the amplitudes of temperature and electric potential are small in the divertor region, as they are roughly proportional to the background plasma temperature. The X-point shear leads to decorrelation of turbulence across the $\mathrm{X}$-point regions, more so for larger toroidal wave numbers.

\section{Acknowledgments}

We would like to acknowledge discussions with Drs. R.H.Cohen, W.M.Nevins, and D.D.Ryutov. This work is performed for USDOE by Univ. Calif. LLNL under contract W-7405- ENG-48 and was supported as LLNL LDRD project 03-ERD-099. 


\section{References}

[1] X. Q. Xu and R.H.Cohen, Contr. Plas. Phys. 36158 (1998).

[2] D.Farina, R.Pozzoli, D.Ryutov, Nuclear Fusion, 331315 (1993).

[3] J.R.Myra et al., Phys. Plasmas 74622 (2000).

[4] X.Q. Xu et al., Phys. Plasmas 71951 (2000).

[5] H.Berk, D.D.Ryutov, Yu.A.Tsidulko, JETP Lett., 5223 (1990).

[6] H.Berk, D.D.Ryutov, Yu.A.Tsidulko, Phys. Fluids, B3 1346 (1991).

[7] A.V.Nedospasov, Phys.Fluids B5, 3191 (1993).

[8] D.D.Ryutov and R.H.Cohen, Contrib. Plasma Phys. 44, No. 1-3, 168 (2004).

[9] M.V.Umansky et al., Contrib. Plasma Phys. 44, No. 1-3, 182 (2004).

[10] S.I. Braginskii, Transport processes in a plasma, Reviews of Plasma Physics, Vol. 1, Ed. M.A. Leontovich (Consultants Bureau, New York, 1965), p. 205.

[11] A.M.Dimits, Phys. Rev. E 48, 4070 (1993).

[12] X. Q. Xu et al., J. Nucl. Mater. 266-269 993 (1999).

[13] A. Mazurenko et al., Phys. Rev. Lett. 8922 (2002).

[14] X.Q. Xu et al., New Journal of Physics 4 53.1-53.15 (2002).

[15] X. Q. Xu et al., Nucl. Fusion 40731 (2000).

[16] X. Q. Xu et al., Nucl. Fusion 4221 (2002).

[17] P.C. Stangeby, "The Plasma Boundary of Magnetic Fusion Devices" Bristol, Institute of Physics Publishing, 2000.

[18] T.D.Rognlien et al., Contr. Plas. Phys. 34362 (1994).

[19] W.M.Nevins, "GKV User's Manual" (unpublished).

[20] R.H.Cohen and D.D.Ryutov, Contr. Plas. Phys. 36161 (1996). 


\section{Figure captions}

Figure 1: Profiles of background plasma density and temperature used in the present calculations. The top figures show the case of poloidally uniform profiles, the bottom figures show the case of poloidally non-uniform profiles with $T_{\text {mid }} / T_{\text {div }} \sim n_{\text {div }} / n_{\text {mid }} \approx 2$. The poloidal grid goes from the inner plate to the outer one. The radial grid goes from the core plasma interface to the outer boundary. The dashed lines show the locations of separatrix and the X-points.

Figure 2: Time-evolution of RMS $<\tilde{\phi}>$ during the initial ramp-up phase. Fluctuations in the divertor legs grow much faster than those at the midplane. The reference gyro-frequency is $\omega_{c i} \approx 7.85 \times 10^{7} \mathrm{rad} / \mathrm{sec}$.

Figure 3: Distribution of RMS $<\tilde{\phi}>$ during the initial ramp-up phase. The fluctuations amplitude is large outside of the separatrix in the outer leg, and in the private flux region in the inner leg. For the chosen profiles of the background plasma these are the regions of unfavorable curvature for ballooning instabilty.

Figure 4: RMS amplitudes of $\tilde{n}_{i}$ plotted against the local $n_{i 0}$ in the inner divertor leg for saturated turbulence. Different plotting symbols show data from five different BOUT cases with same background plasma parameters at the mid-plane, $n_{i} \approx 1.2 \times 10^{13} \mathrm{~cm}^{-3}$ and $T_{e, i} \approx 50 \mathrm{eV}$, and divertor parameters as indicated in the figure, where temperatures are in $\mathrm{eV}$, densities in $10^{13} \mathrm{~cm}^{-3}$

Figure 5: RMS amplitudes of $\tilde{\phi}_{i}$ and $\tilde{T}_{e}$ plotted against the local $T_{e 0}$ in the inner divertor leg for saturated turbulence. 
Figure 6: The cross-correlation strength is plotted against the poloidal grid index for pertubations with three different toroidal wavenumbers. The cutoffs of the cross-correlation at the X-points are stronger for larger toroidal wavenumber, $k_{\phi}$. 

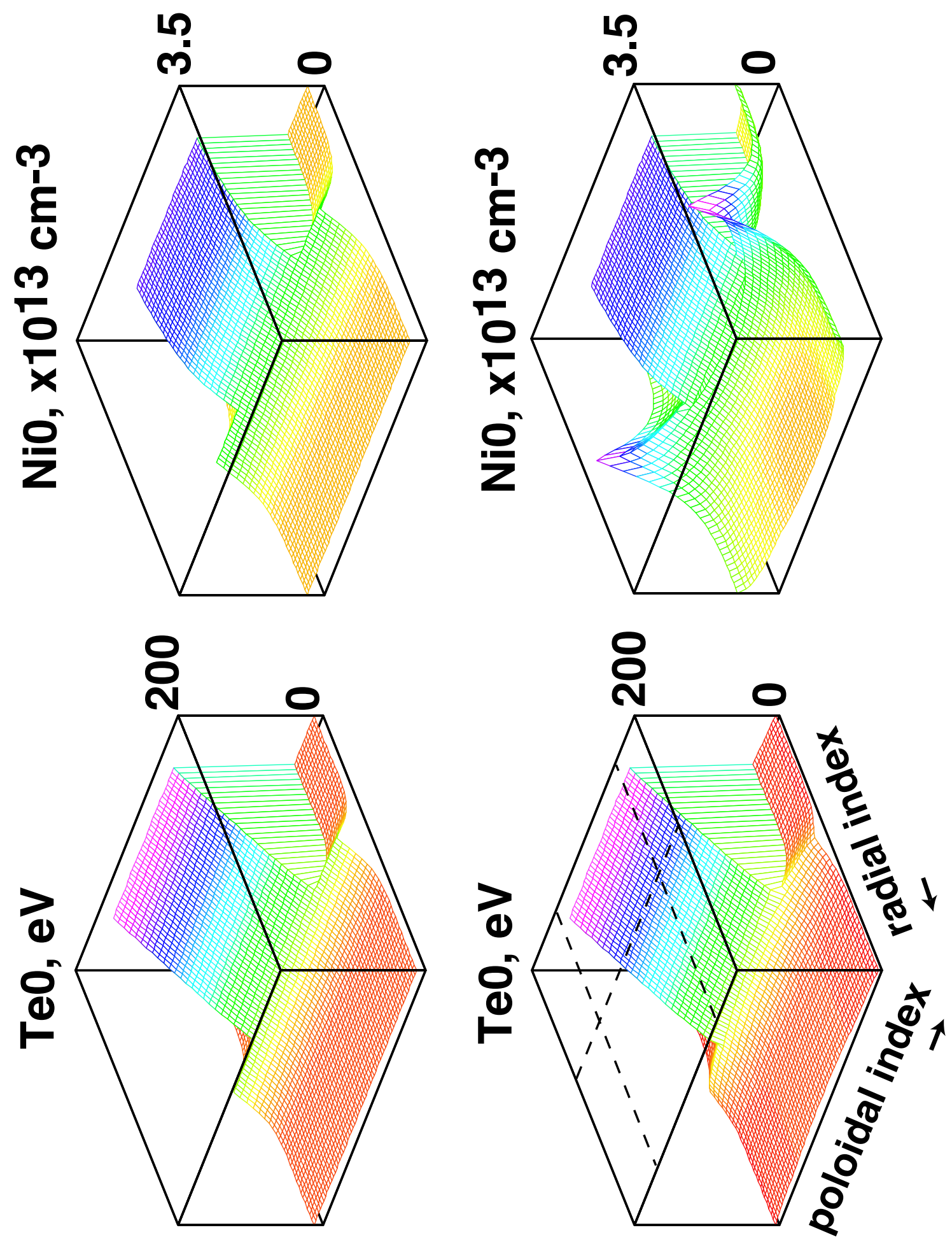


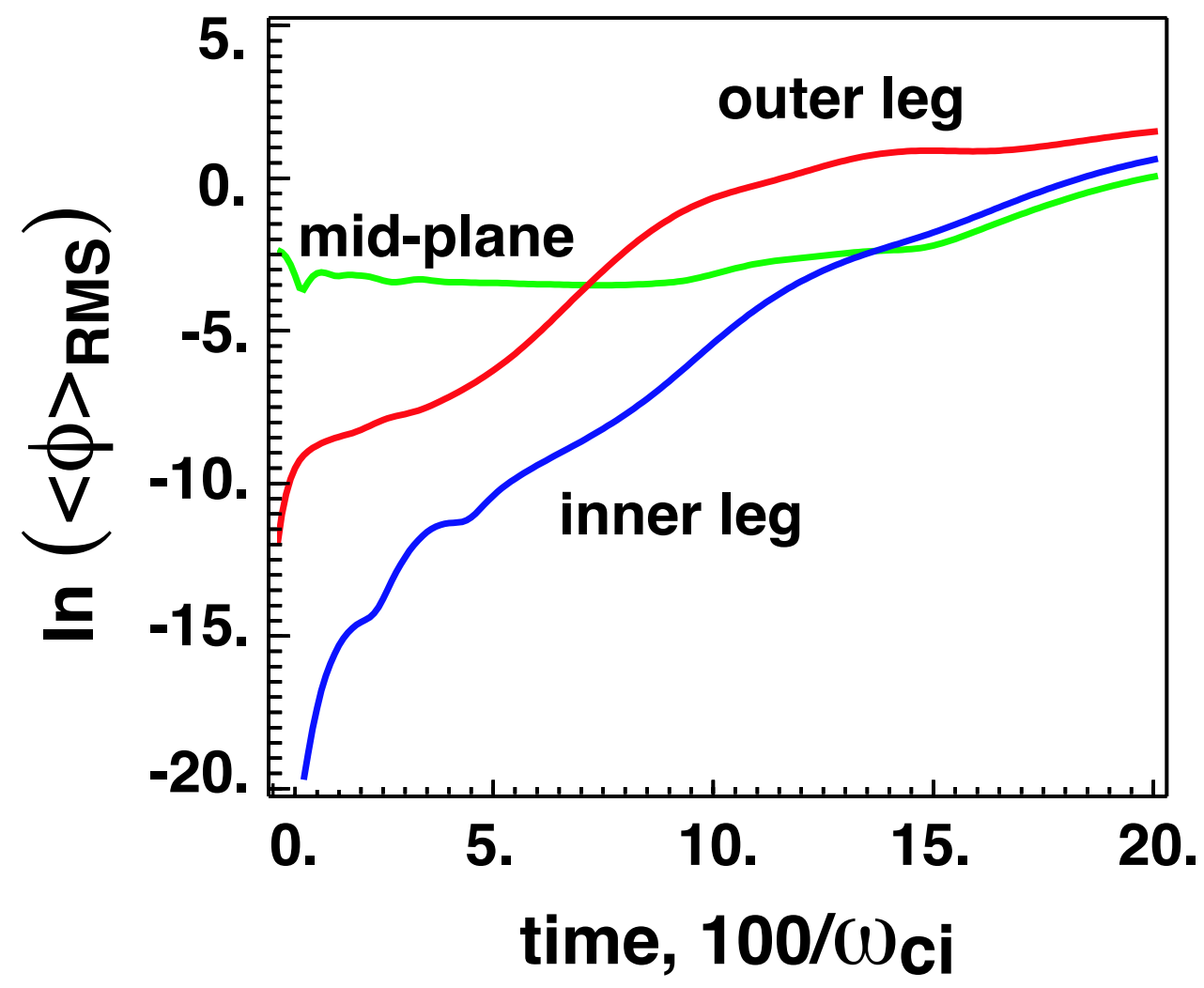

Fig. 2 


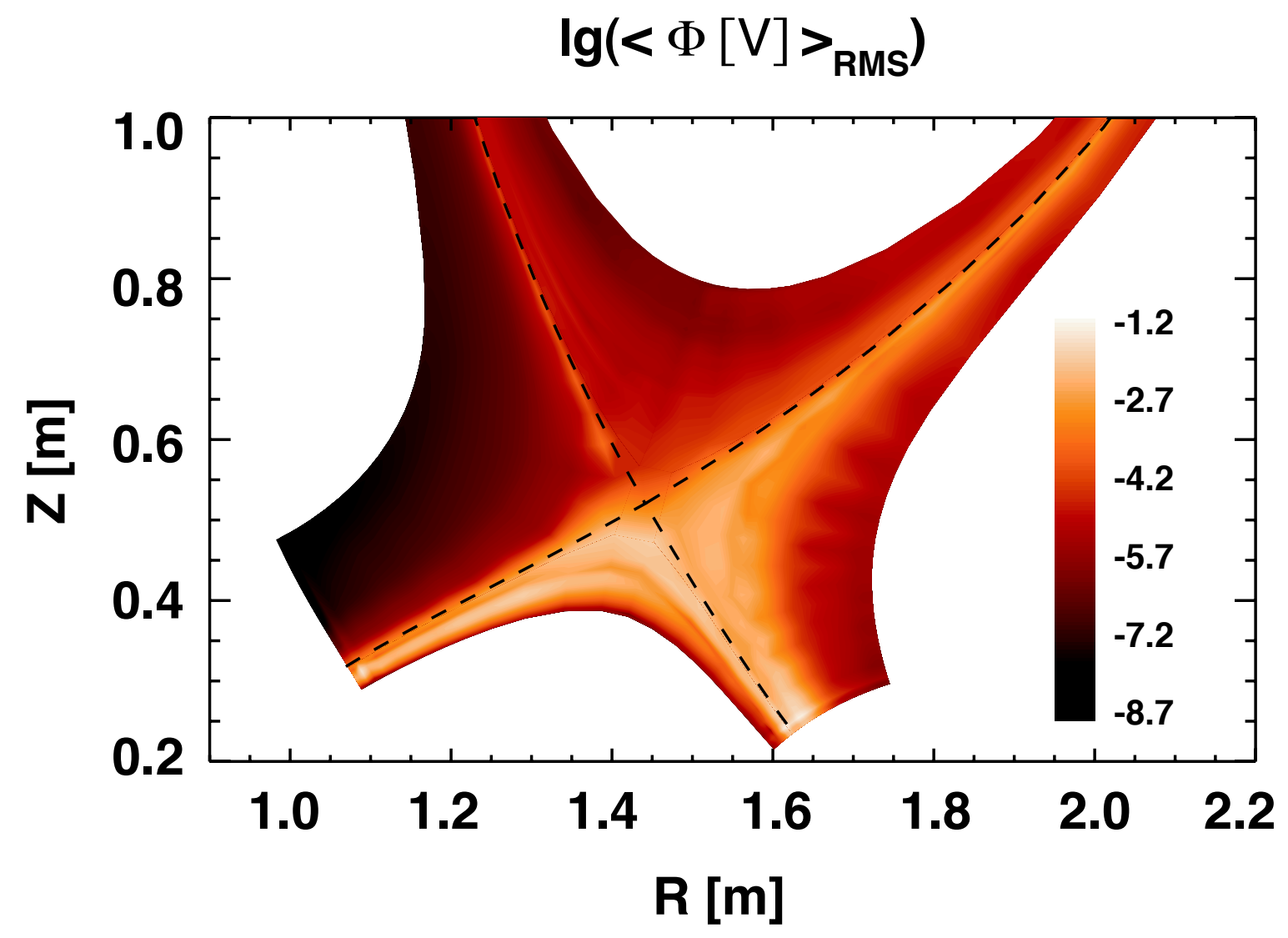

Fig. 3 


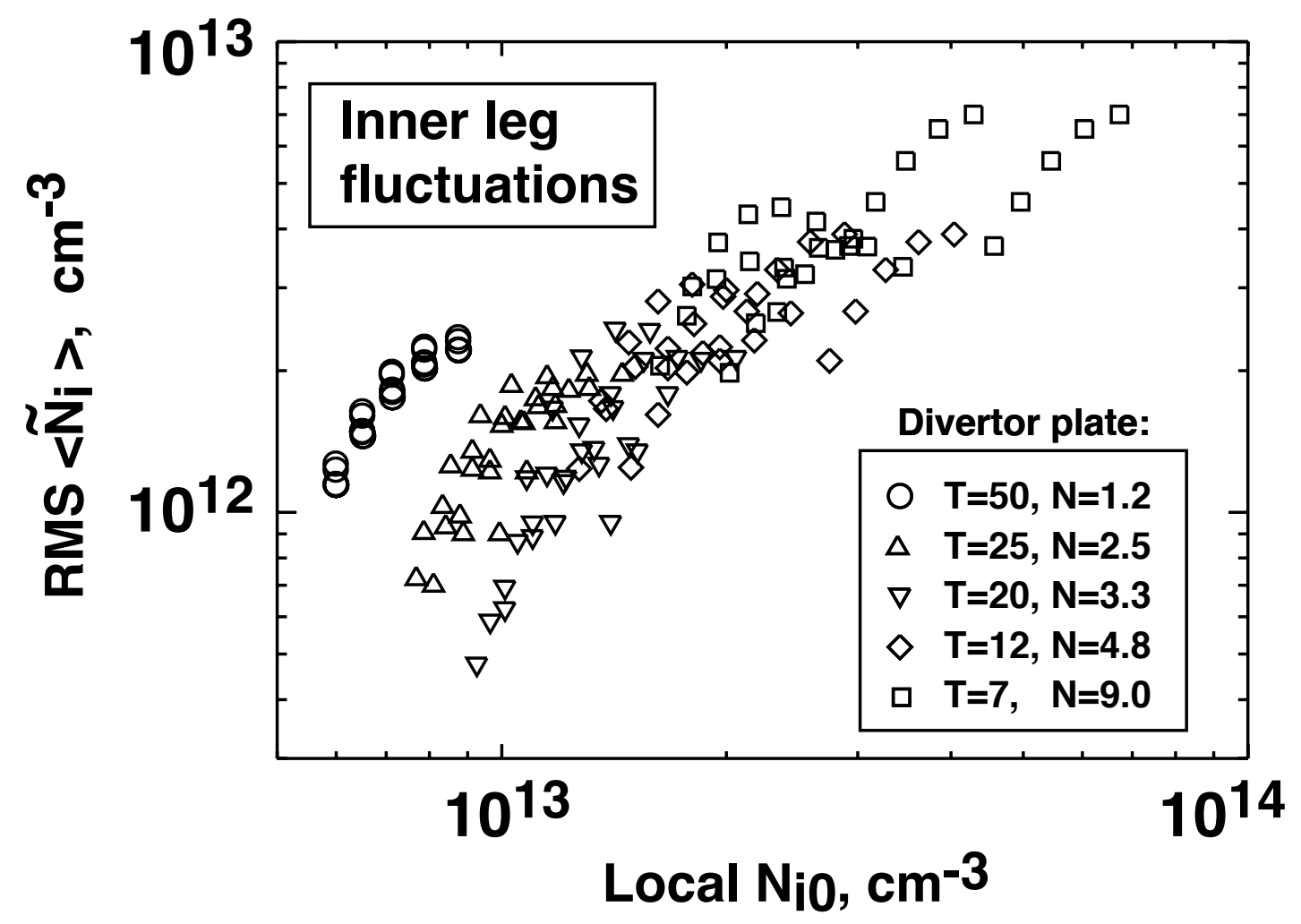

Fig. 4 

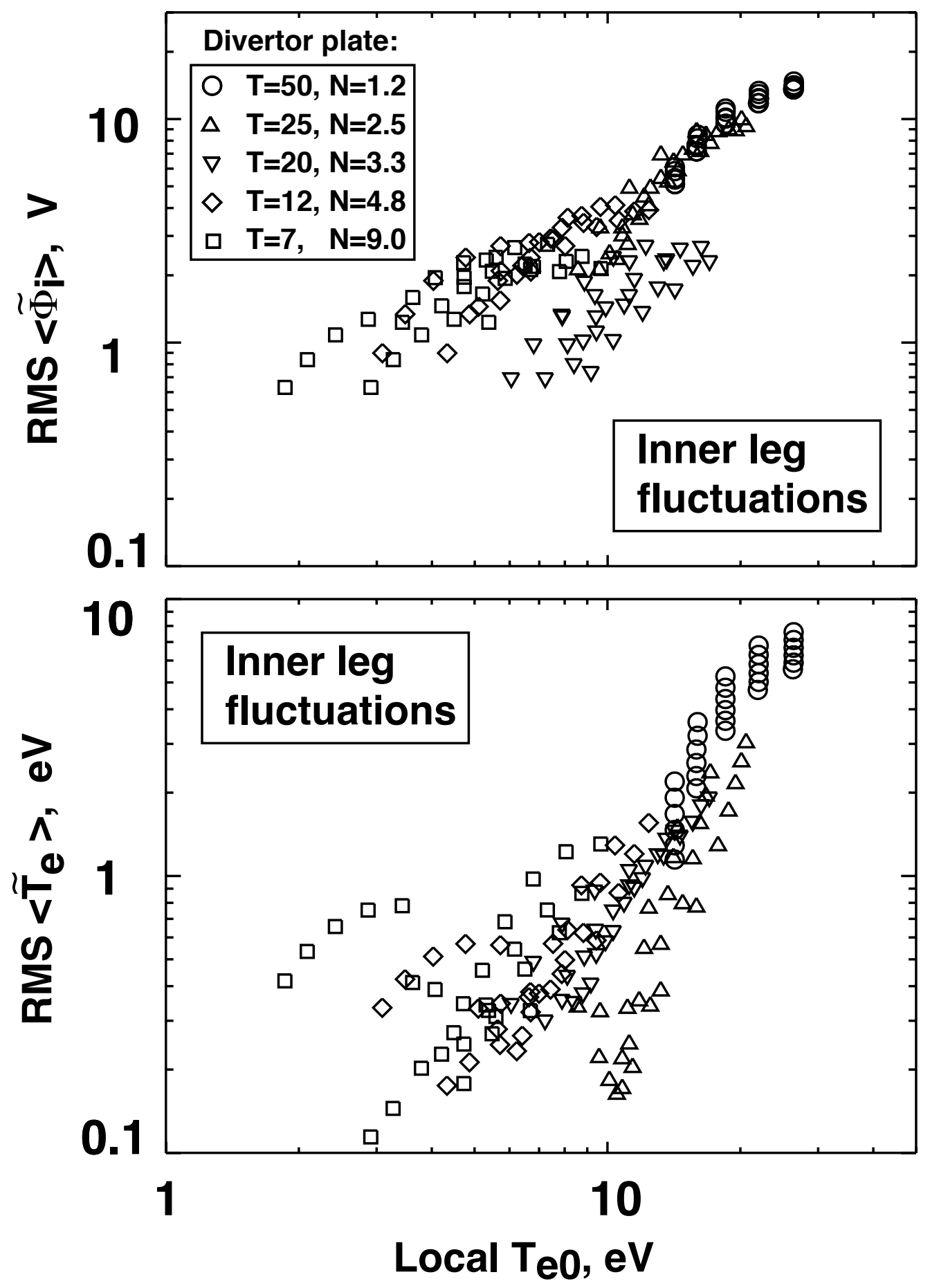

Fig. 5 


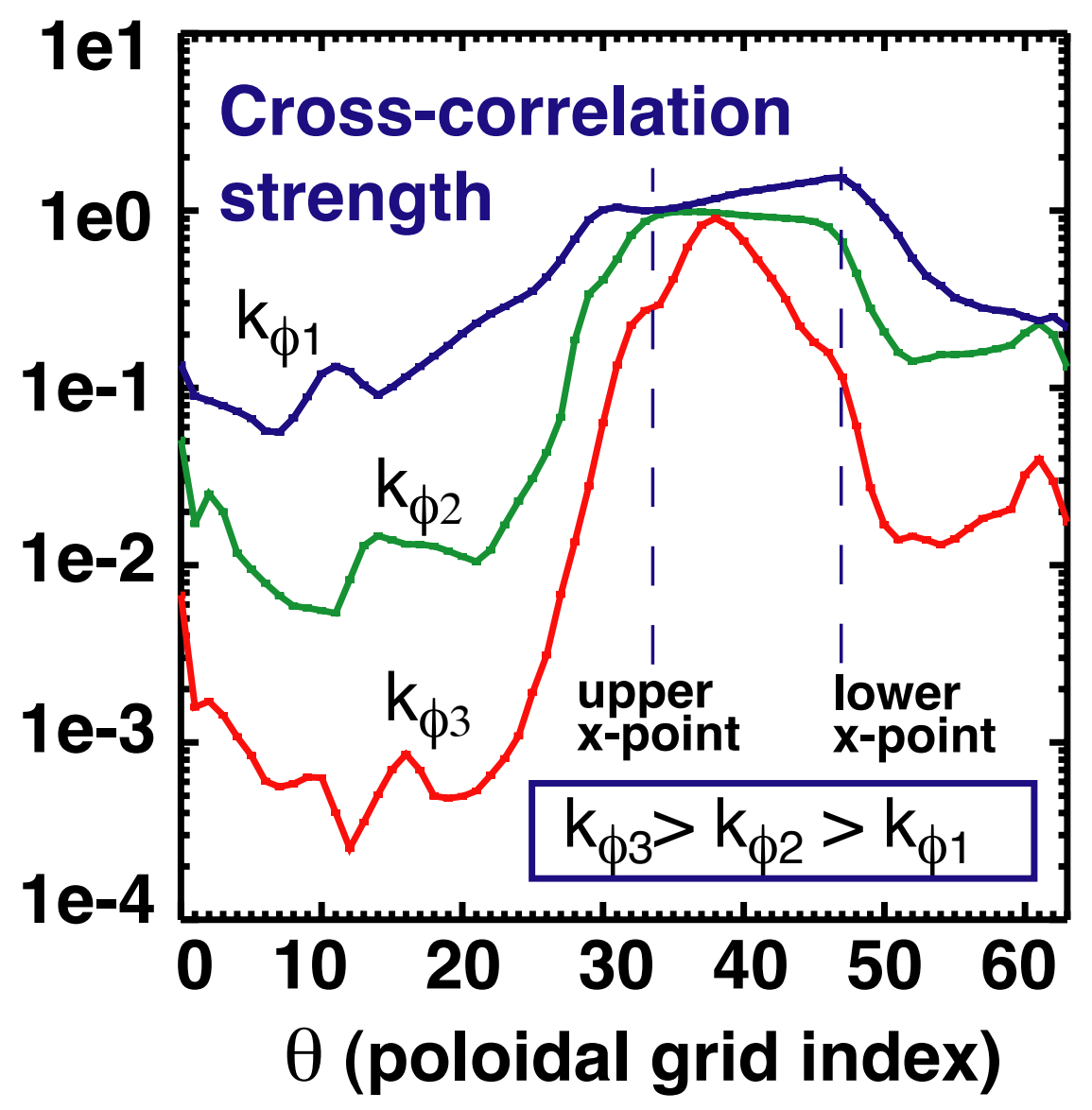

Fig. 6 